\title{
THE KITT PEAK MAGNETOGRAPH
}

\section{IV: 40-Channel Probe and the Detection of Weak Photospheric Fields}

\author{
W. LIVINGSTON and J. HAR VEY
}

Kitt Peak National Observatory*, Tucson, Arizona, U.S.A.

\begin{abstract}
The 40-channel magnetograph is described. Special attention is given to details of the fiber-optic probe which dissects the Fraunhofer line image and provides for a choice of spectral and spatial resolution. Examples of magnetograms taken with the instrument are shown. Maps of quiet areas, made with long integration times to achieve a noise of $0.4 \mathrm{G}$, yet with 5 arc-sec resolution, indicate the presence everywhere of a minimum background field of average strength 2-3 G.
\end{abstract}

\section{Introduction}

We describe here a new instrument of the Babcock-type, intended to achieve high resolution in magnetic observations without a sacrifice of limiting sensitivity. The number of spatial elements that are simultaneously observed has been increased 40-fold. Among other problems, the instrument is being applied to the study of the fine structure of weak fields.

The resolution realized with the photoelectric magnetograph depends on the size of the exploring aperture, the seeing, and the signal/noise ratio (which in turn is proportional to the square root of the light flux). As the aperture is made small, the order of one arc s or less, the flux is reduced and to maintain a given $\mathrm{S} / \mathrm{N}$ the scan speed must be slower, a circumstance unfavorable for good resolution because of atmospheric seeing. Beckers (1968) has reviewed these conditions and concluded that for high resolution, photographic techniques are superior to single-channel photoelectric magnetographs. We agree with Beckers but have sought to improve our resolution by expanding our magnetograph from the single aperture to a linear array of 40 , thus creating something like a photoelectric spectroheliograph.

\section{The Fiber-Optic Probe}

Figure 1 diagrams the essential optical components as positioned on the spectrograph. In front of the entrance slit is the analyzer for circularly polarized light; a KDP crystal (ISOMET, Inc.) having a clear aperture of $3 \times 50 \mathrm{~mm}$ followed by a polaroid. The spectrograph slit is set in width to $1, \frac{1}{2}$, or $\frac{1}{4} \mathrm{~mm}$ corresponding to approximately 2 , 1 , or $\frac{1}{2}$ arc s on the incident solar image.

In the camera plane of the $13.5 \mathrm{~m}$ Czerny-Turner spectrograph (FL collimator= FL camera) are two fiber-optic probes which serve as double exit slits along the direction of dispersion. One of the probes is equivalent to a rather broad set of exit slits $(0.175-0.150-0.175 \AA$ in our grating 5 th order $)$ and provides a match to the

* Operated by the Association of Universities for Research in Astronomy, Inc., under contract with the National Science Foundation. 
wings of the strong line of Fe I $\lambda$ 5233. Harvey and Livingston (1970) have calculated the magnetograph response signal resulting from this line and slit arrangement to be linear $( \pm 1 \%)$ up to fields of $4000 \mathrm{G}$ (paper II). Fortunately, the wings of Fe I $\lambda 5233$ are relatively insensitive to temperature changes so this line and probe provide wide dynamic range and, we believe, an accurate indication of the line of sight field.

The second probe is appropriate for a much narrower line such as Fe I $\lambda 5250$

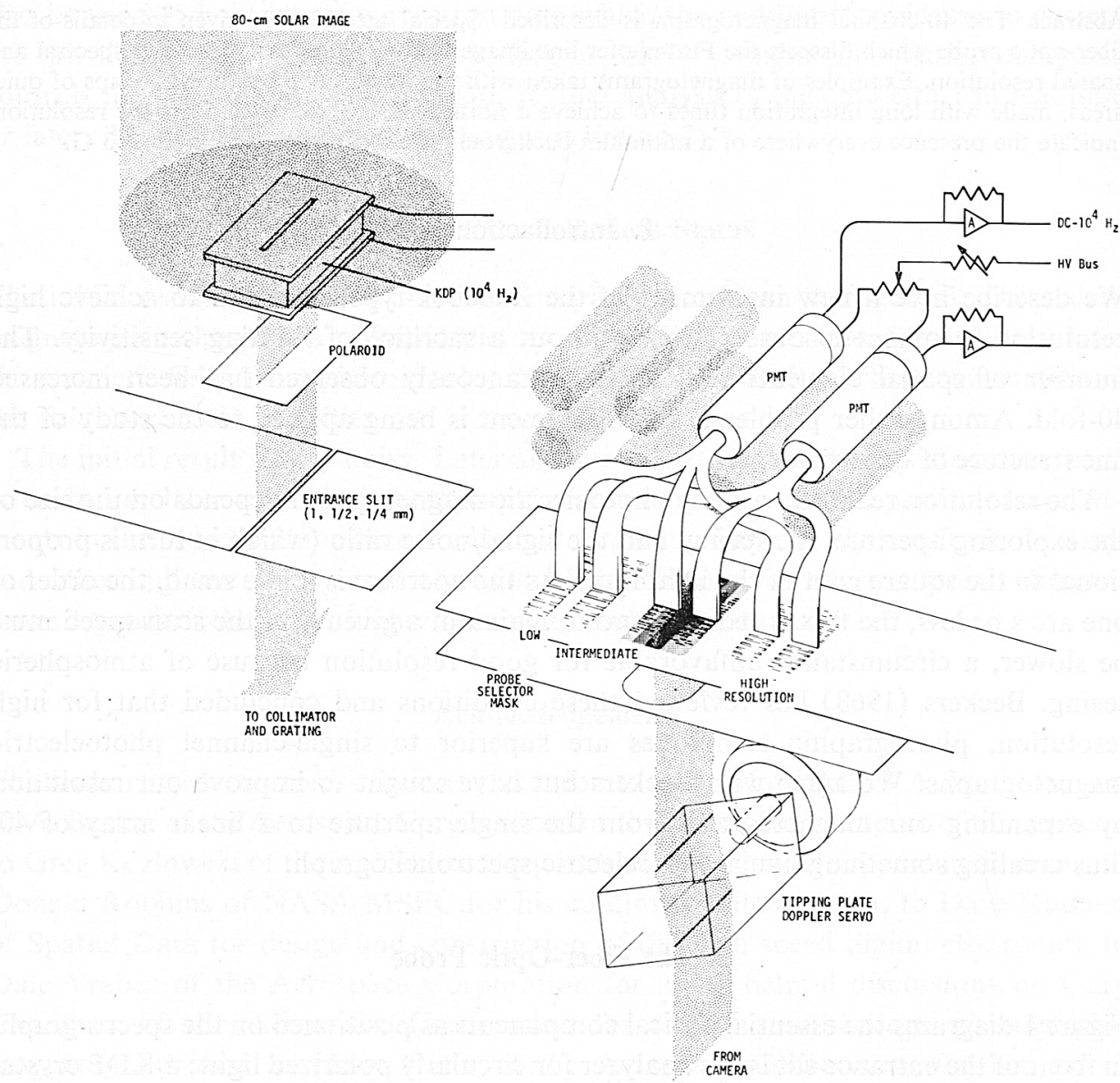

Fig. 1. Arrangement on the spectrograph. Not shown is a second probe with parallel light feed to the PMTs. The tipping plate Doppler compensating servo is controlled by summed signals from all 40 (20) channels.

$(0.067-0.028-0.067 \AA)$. For the detection of weak fields this line and probe provide a $\mathrm{S} / \mathrm{N}$ about twice that obtained with $\mathrm{Fe} \mathrm{I} \lambda 5233$, but there is some uncertainty in the measurements because of the variable profile of Fe I $\lambda 5250$ (Chapman and Sheeley, 1968; Harvey and Livingston, 1969).

Each of the above spectral probes is subdivided into three sizes of spatial sections, 


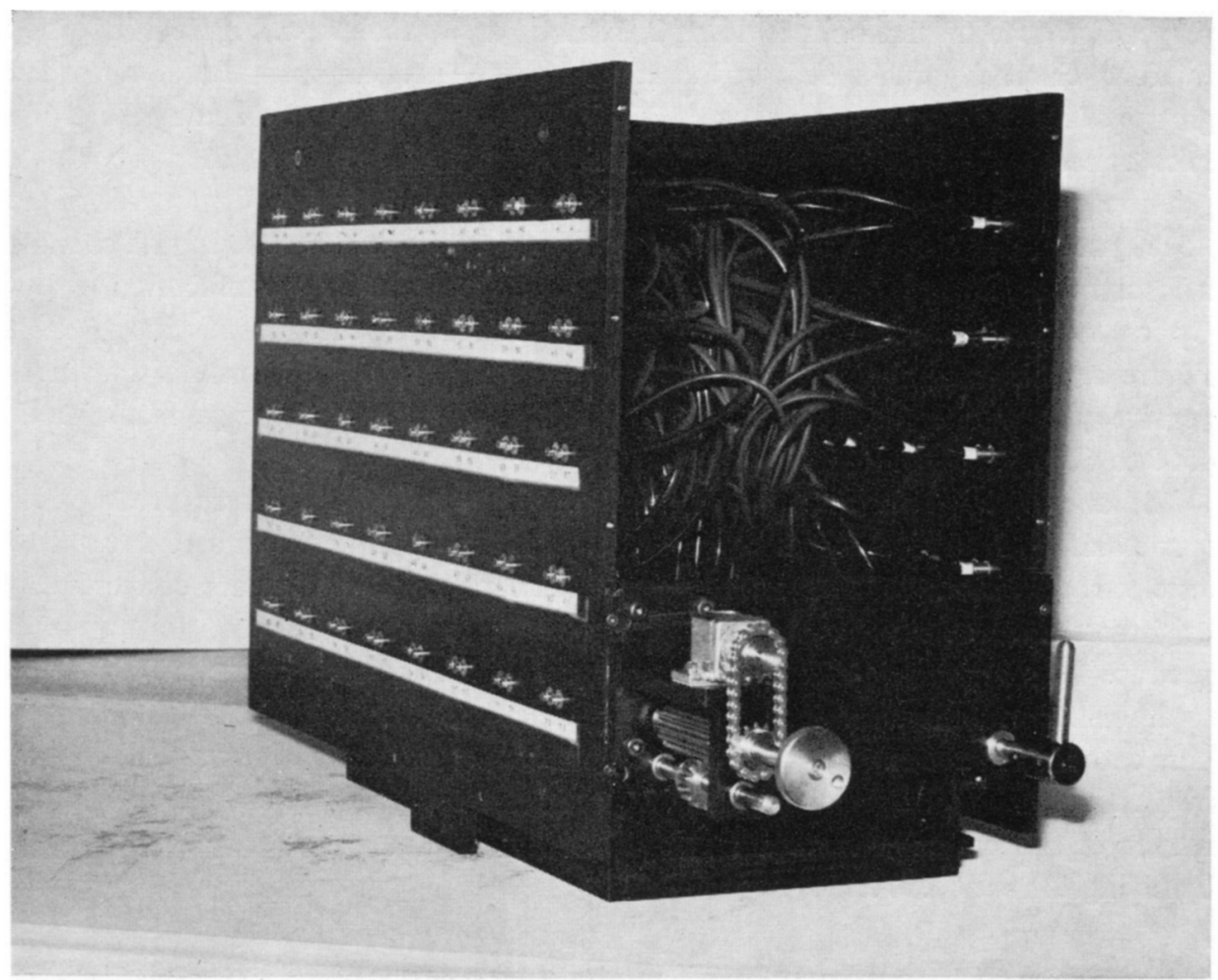

Fig. 2. Pre-assembly photo of the fiber optic probe unit. The knob with chain drive to a counter translates the $\mathrm{Fe}$ I 5233 probe with respect to $\mathrm{Fe}$ I 5250 . The right hand control moves both probes together over the 'probe selector mask'.

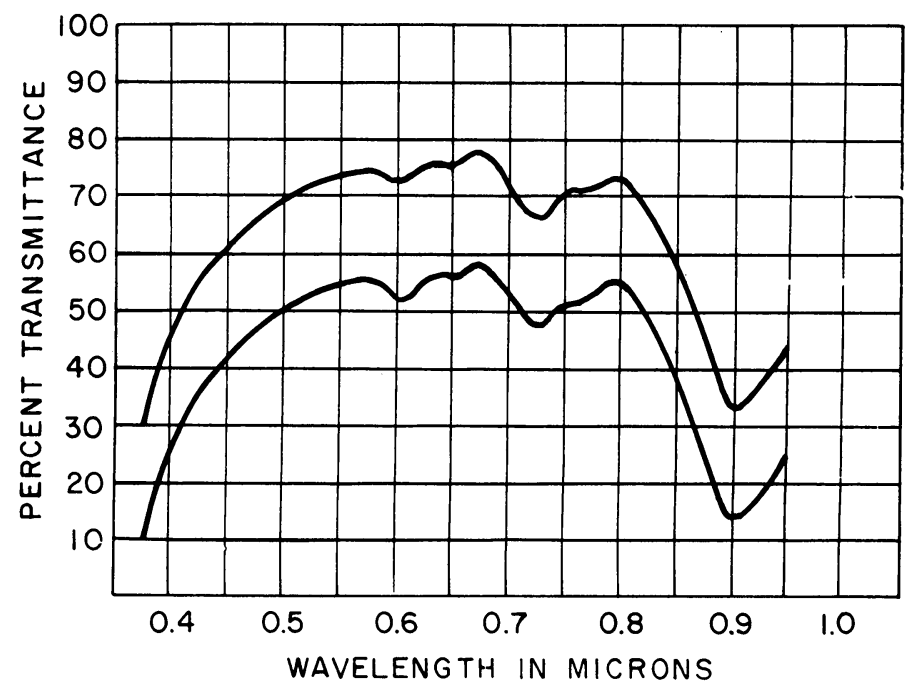

Fig. 3. Transmission of $30 \mathrm{~cm}$ length of plastic fiber (length employed $\leqslant 30 \mathrm{~cm}$ ). Upper curve assumed no end losses, i.e. optical contact to source-receiver. Lower curve includes end losses. 
perpendicular to dispersion, providing 2,1 , and $\frac{1}{2}$ arc s resolution. Figure 1 indicates the forty channel parallel feed for the $\lambda 5233$ probe. There are a total of six possible groups of fibers converging on each PMT. The group in use is controlled by the probe selector mask. Additionally, there is provision for masking off half the groups of a given resolution, translating the $\lambda 5233$ probe sideways into spatial alignment with the $\lambda 5250$ probe, and thus obtain simultaneous 2 spectral channel and 20 spatial channel measurements. Figure 2 shows the probes, which were custom manufactured (PolyOptics, Inc.). They are made from a plastic material having the transmission given in Figure 3. This transmission, combined with the S-10 spectral response of our inexpensive photomultiplier tubes (EMI 9524C), limit spectral coverage from about 4500 to $6000 \AA$.

The anode signal from each PMT is passed through a miniature preamplifier (bandwidth DC- $10^{4} \mathrm{~Hz}$ ) located on the tube socket, then routed over a $10 \mathrm{~m}$ cable to an electronics rack. The DC components from each pair of tubes are converted to brightness and velocity signals. The AC component is phase-detected at $10^{4} \mathrm{~Hz}$ to produce the magnetic signals. These three signals are sequentially sampled, converted to digital information, and recorded on magnetic tape. This data tape is used to prepare cathode-ray tube pictures, contour maps or other representations of the mapped fields. Further details concerning the instrument as a polarimeter (paper I), data reduction, calibration procedures (II), and display techniques (III) are given in papers by Livingston et al., 1970.

\section{Preliminary Results on Solar Fields}

Two kinds of observations are being taken with the 40-channel system: Full Disk Maps of low resolution which are taken on a semi-routine basis, and Area Scans which cover smaller regions for special projects with possibly higher resolution and sensitivity.

\section{FULL DISK MAPS}

Using the low resolution Fe I $\lambda 5233$ probe, the entire solar image is mapped in 21 swaths in $40 \mathrm{~min}$. The number of recorded elements on the disk is $6\left(10^{5}\right)$. A time constant of $33 \mathrm{~ms}$ has been chosen to optimize the spatial resolution. The lineof-sight field strength measured ranges from the noise level $(\sim 15 \mathrm{G})$ to $3000 \mathrm{G}$. The spatial resolution is most often determined by image motion or 'seeing'. Beginning February 1970 an average of 3 full disk magnetograms have been obtained each month. We expect to continue and enlarge this program as telescope time allows.

A great utility to these full disk records is that the region of interest need not be anticipated in advance. For example, on the record for 7 March, 1970 (Figure 4) the rather insignificant region just off the far south meridian has proven interesting in the interpretation of nearly simultaneous X-ray pictures (Van Speybroeck et al., 1970). 




Fig. 4. Full disk magnetogram of 7 March, 1970 taken in Fe I 5233. Fields of positive polarity are indicated dark, negative light.

\section{AREA SCANS}

In this observing mode the image is step scanned. For each step the image is held fixed while the computer samples and integrates the signals from the 40 channels. After this integration period, preselected from 0.015 to $16^{s} 0$, the image is stepped one resolution element and the process repeated.

Figures 5 through 7 afford a comparison of the full disk and area scan results. Figure 5 is a full disk brightness map made in $\mathrm{Fe}$ I $\lambda 5250$ under relatively poor seeing. Figure 6 is an area scan of the region west of the central meridian taken the following morning under good seeing conditions. The low resolution probe was employed for 


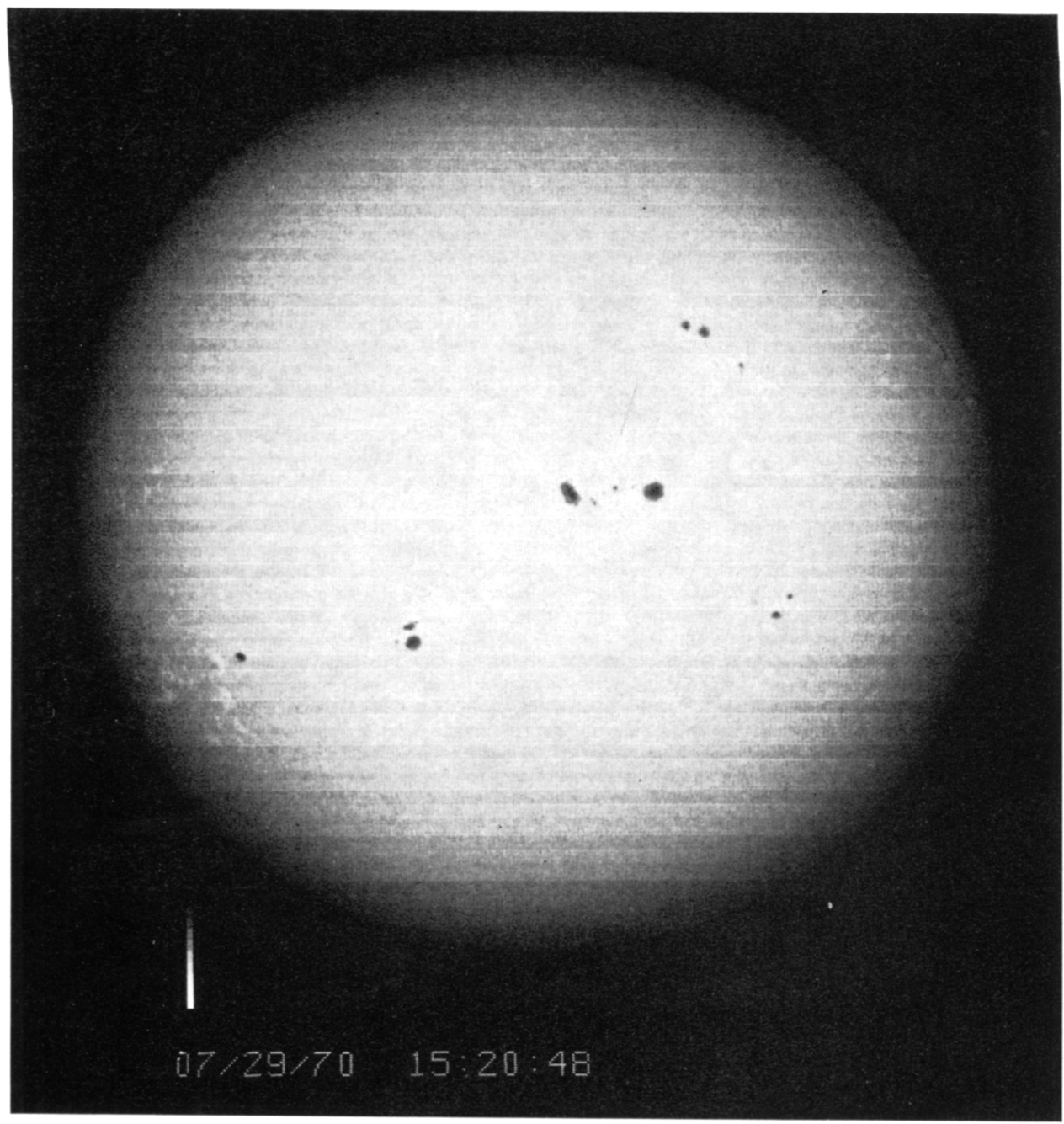

Fig. 5. Brightness record for the full disk map of $29 \mathrm{July}, 1970$ at 15:20 local time. North is top, West is right. Resolution is seeing limited.

both the observations but while the full disk observation is seeing limited, the area scan record is probe limited. Finally, Figure 7 is a four-swath scan of this same region with the $\frac{1}{2}$ arc s probe demonstrating an achieved resolution comparable to that obtained with photographic methods.

\section{Measurements of Weak Fields}

Compared with photographic techniques the 40-channel system can detect much weaker fields. Fe I $\lambda 5250$ area scans made with a long integration time of $16 \mathrm{sec}$ yield 


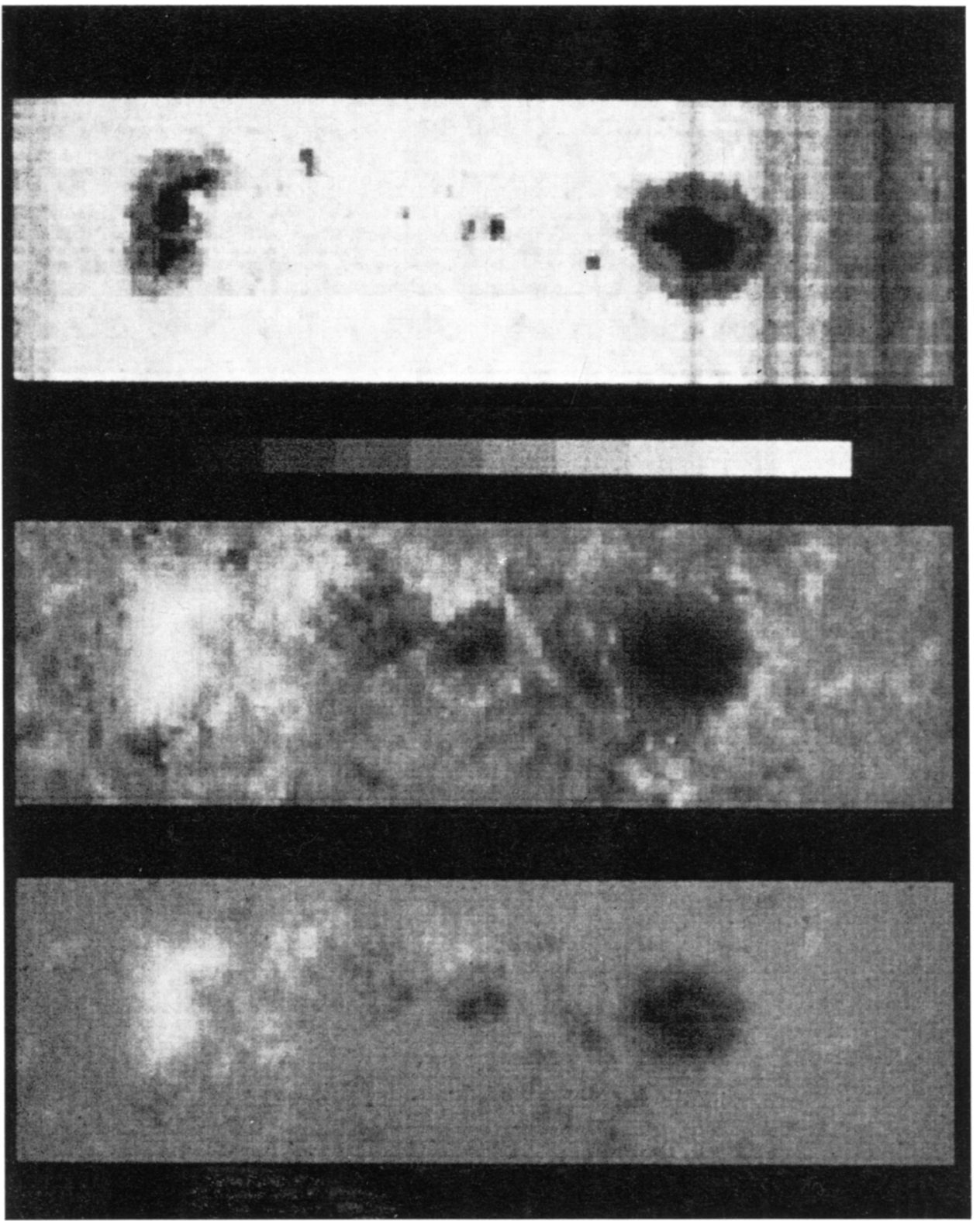

Fig. 6. Area scan of the region near the center of Figure 5 taken on 30 July, 1970 at $07: 35$ local time under good seeing conditions. Resolution is limited by the 2 arc s probe. Top frame is the brightness; a thin cloud produced the streaks near the right edge. In the bottom frame the departure from grey is proportional to the field strength and in the middle frame to the square root of the field strength to emphasize weak fields. 

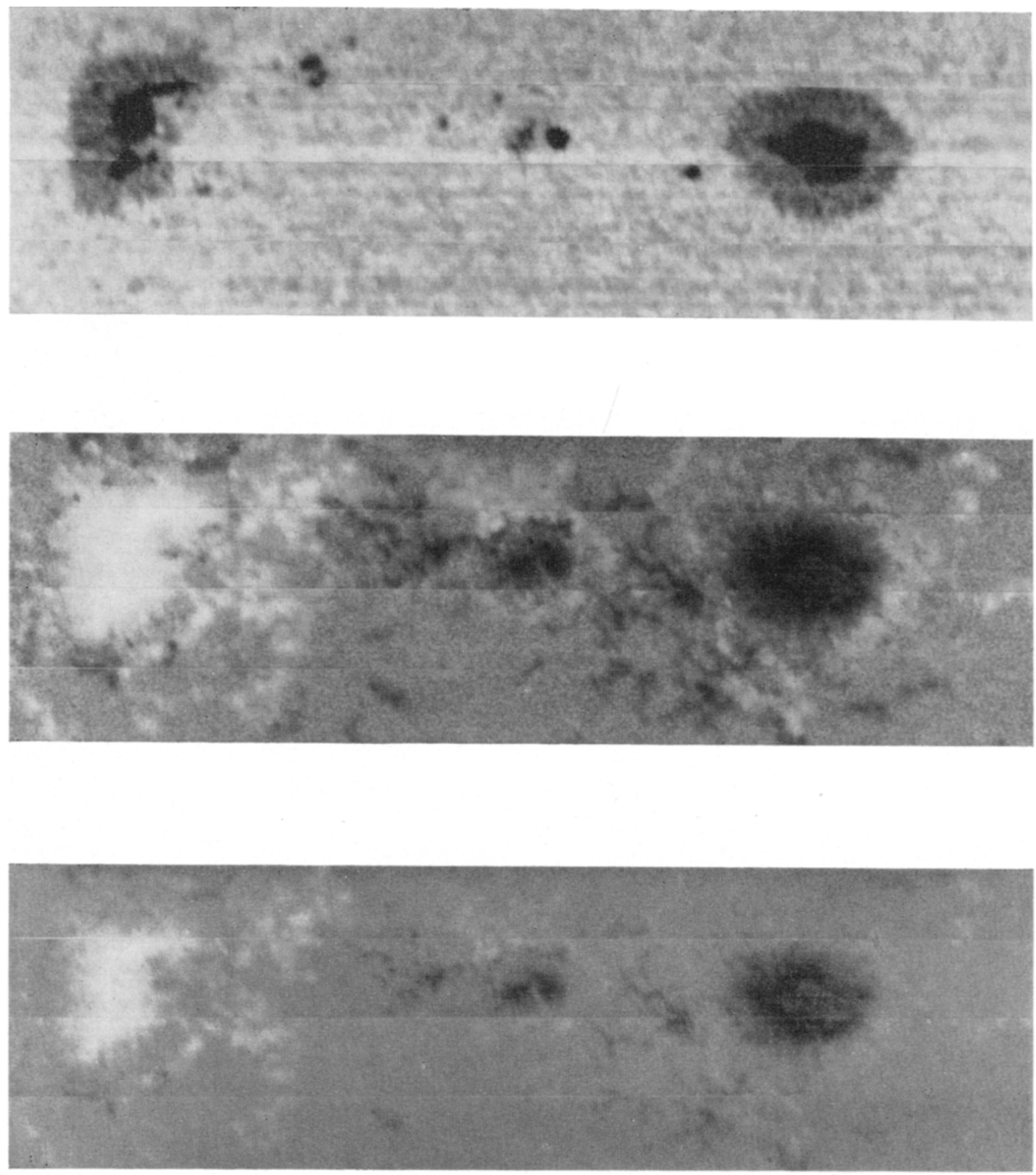

Fig. 7. Same as Figure 6 except with $\frac{1}{2}$ arc s probe. Now the spatial resolution is again seeing limited.

maps of good resoution ( $\sim 5$ arc s) yet with a noise of about $0.4 \mathrm{G}$. The combination of good resolution and high sensitivity permits us to discriminate between the well known small regions with strong magnetic fields and possible large-scale areas with weak magnetic fields. Preliminary observations indicate that between the strong fields a weak field is found everywhere. Assuming that the normal disk profile of $\mathrm{Fe} \mathrm{I}$ $\lambda 5250$ holds for these weak field regions, the average field strength is 2-3G.

Evidence for the weak fields is contained in Figures 8-10. We have plotted the 
isogauss contours for fields stronger than $20 \mathrm{G}$ which are concentrated in small areas. Below $20 \mathrm{G}$ only the polarity is displayed as light and dark shading. The pattern of data elements is visible in this shading. Except for a very local coherence extending over about 5 arc $s$ caused by the seeing, the data sample elements are independent. The areas of like polarity are seen invariably to extend over many data elements. We take this coherence of polarity over extended areas as evidence for the weak but

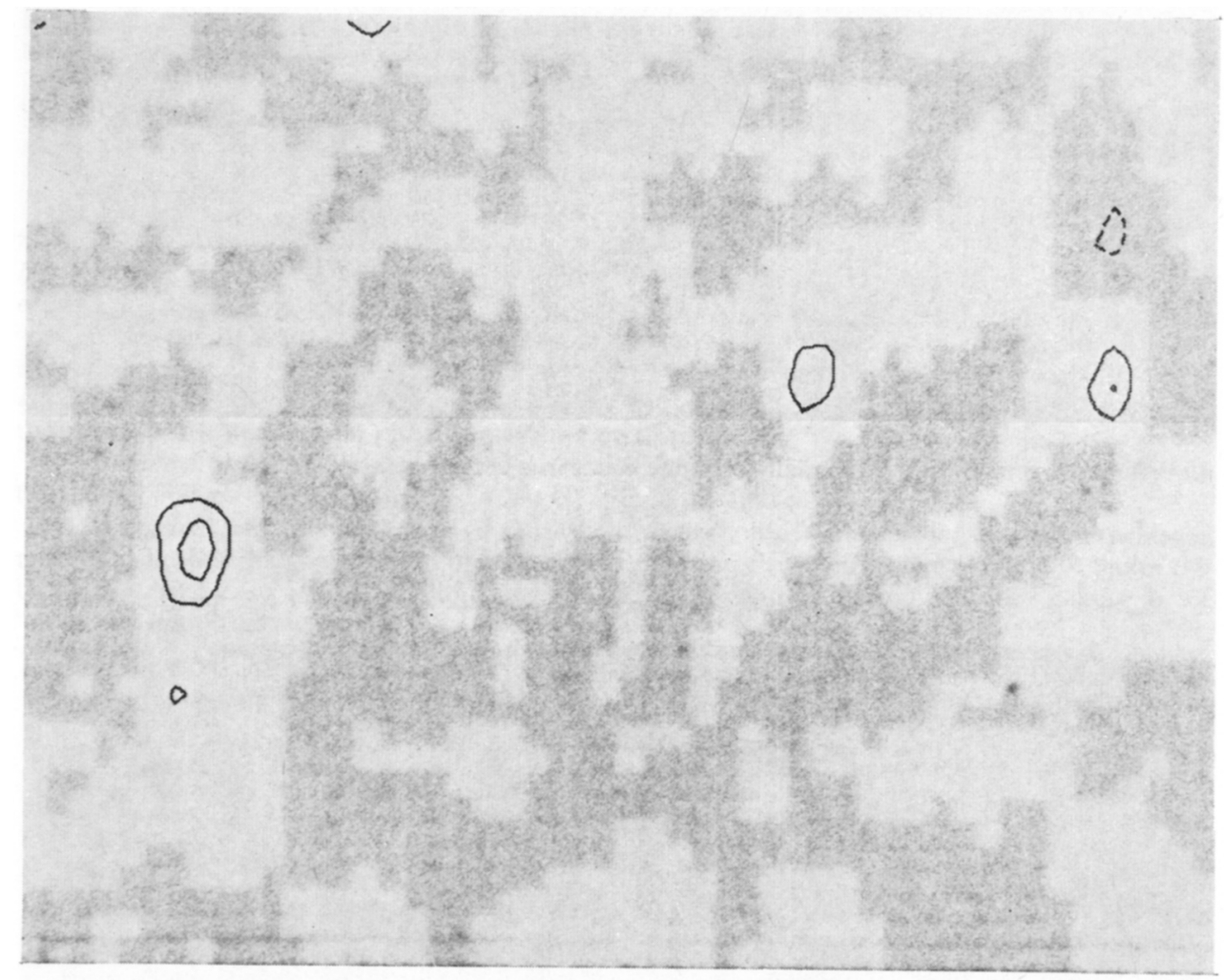

Fig. 8. Area scan with $16 \mathrm{~s}$ time constant, $\mathrm{Fe}_{\mathrm{I}} 5250,2$ arc $\mathrm{s}$ probe, of high latitude quiet region. Contour intervals are 20, 40,.. G. Noise $\sim 0.4$ G. Shading indicates polarity of weaker, non-contoured fields. Contiguous character of these polarity indicators is evidence for a large scale weak field.

significant background field. Figure 10 is a recording identical in every way to the preceding except taken with the KDP off. The pattern produced is essentially random. However, it is well to note that this does not prove that the pattern seen when the modulation is on is actually due to a weak background field. Whenever one measures weak magnetic fields with a Babcock magnetograph it is important to make sure that there is no instrumental elliptical polarization. When such polarization is present, a small Doppler shift can masquerade as a weak magnetic field. Since we can only 


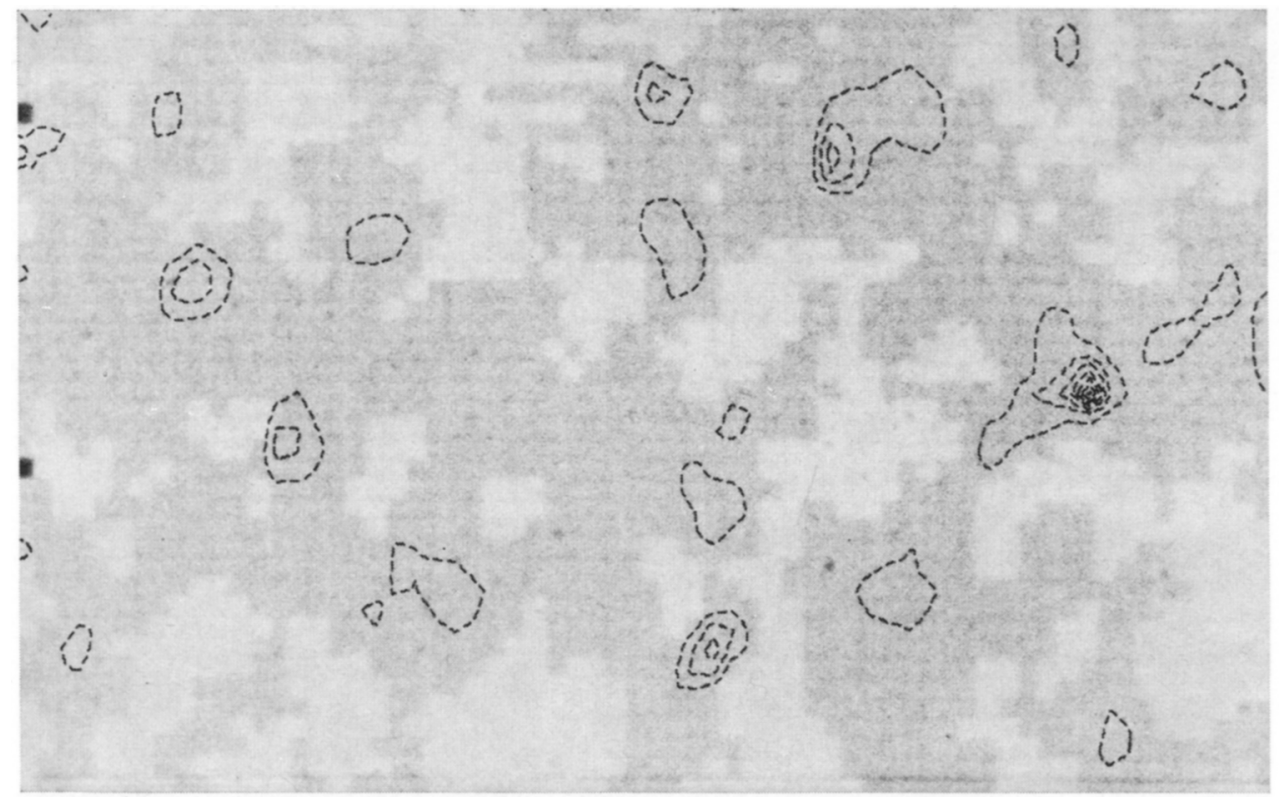

Fig. 9. Similar to Figure 8 but in opposite hemisphere.

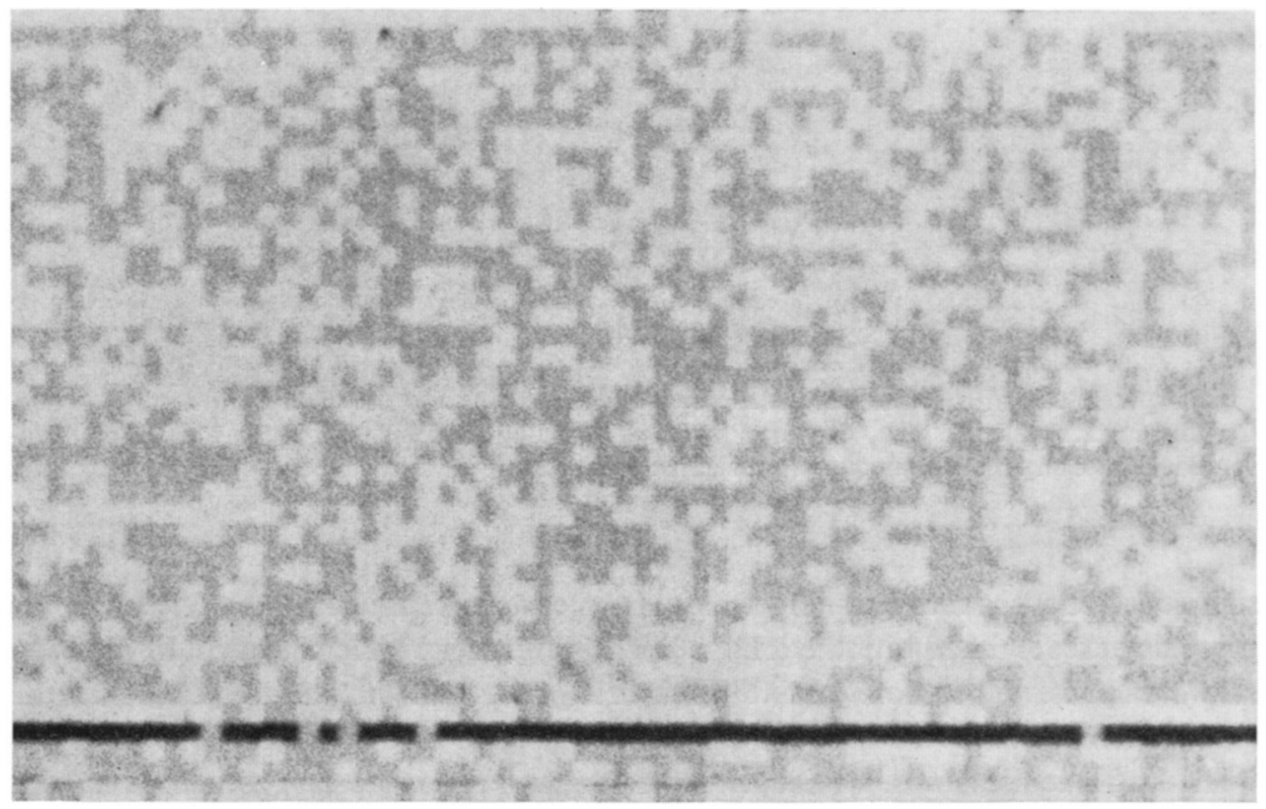

Fig. 10. Same as Figure 9 except with KDP off, showing random polarity pattern for comparison. One channel was erratic and has been deleted from the diagram. 
correct the average Doppler shift of the 40 spatial channels it is possible that the weak field we see is just the pattern of velocity fields present. Further observations are in progress.

\section{References}

Beckers, J. M.: 1968, Solar Phys. 5, 15.

Chapman, G. A. and Sheeley, N. R. Jr.: 1968, Solar Phys. 5, 442.

Harvey, J. and Livingston, W.: 1969, Solar Phys. 10, 283.

Livingston, W. and Harvey, J.: 1971, (paper I) submitted to Solar Phys.

Harvey, J. and Livingston, W.: 1971, (paper II) submitted to Solar Phys.

Livingston, W., Harvey, J., and Slaughter, C.: 1970, (paper III) IAU Colloquium No. 11, Automation in Optical Astrophysics, Edinburgh, 12-14 August, 1970.

Van Speybroeck, L., Krieger, A., and Vaiana, G.: 1970, Nature 227, 818.

\section{Discussion}

$N$. R. Sheeley, Jr.: This is going to be a dirty question. Would you care to comment on the existence of magnetic quanta?

$W$. Livingston: It seems likely we are observing three rather different kinds of fields on the Sun: sunspot associated fields, strong but non-sunspot small-scale fields (your 'gaps'), and now these diffuse very weak fields. Any notion of a single-valued or quantized characteristic would apply only to the gap fields. But I would rather not expand on this here.

$G$. W. Simon: What are the typical field strengths of the contoured strong fields when you integrate for $16^{s}$ and find a weak background field 2-3 G? (Figures 8-10).

$W$. Livingston: They range 20 to $140 \mathrm{G}$ in the sample studied. One must realize that the achieved resolution in these long integration observations is not nearly as good as that shown in Figures 6-7 because of seeing, so I would avoid a quantitative interpretation. Presumably they belong to the "magnetic knot" or gap category. 